\title{
BMJ Open The contribution of primary prevention medication and dietary change in coronary mortality reduction in England between 2000 and 2007: a modelling study
}

\author{
M Guzman-Castillo, ${ }^{1}$ R Ahmed, ${ }^{1}$ N Hawkins, ${ }^{2}$ S Scholes, ${ }^{3}$ E Wilkinson, ${ }^{4} \mathrm{~J}$ Lucy, ${ }^{5}$ \\ S Capewell, ${ }^{1}$ M O'Flaherty ${ }^{1}$
}

To cite: Guzman-Castillo M, Ahmed R, Hawkins N, et al. The contribution of primary prevention medication and dietary change in coronary mortality reduction in England between 2000 and 2007: a modelling study. BMJ Open 2015;5:e006070. doi:10.1136/bmjopen-2014006070

- Prepublication history and additional material is available. To view please visit the journal (http://dx.doi.org/ 10.1136/bmjopen-2014006070).

Received 14 July 2014 Revised 28 October 2014 Accepted 6 November 2014

CrossMark

For numbered affiliations see end of article.

Correspondence to Dr M Guzman Castillo; mdlgc106@liverpool.ac.uk

\section{ABSTRACT}

Objective: To analyse the falls in coronary heart disease (CHD) mortality in England between 2000 and 2007 and quantify the relative contributions from preventive medications and population-wide changes in blood pressure (BP) and cholesterol levels, particularly by exploring socioeconomic inequalities.

Design: A modelling study.

Setting: Sources of data included controlled trials and meta-analyses, national surveys and official statistics.

Participants: English population aged $25+$ in 20002007.

Main outcome measures: Number of deaths prevented or postponed (DPPs) in 2007 by socioeconomic status. We used the IMPACT SEC $_{\text {model }}$ which applies the relative risk reduction quantified in previous randomised controlled trials and metaanalyses to partition the mortality reduction among specific treatments and risk factor changes.

Results: Between 2000 and 2007, approximately 20400 DPPs were attributable to reductions in BP and cholesterol in the English population. The substantial decline in BP was responsible for approximately 13000 DPPs. Approximately 1800 DPPs came from medications and some 11200 DPPs from populationwide changes. Reduction in population BP prevented almost twofold more deaths in the most deprived quintile compared with the most affluent. Reduction in cholesterol resulted in approximately 7400 DPPs; approximately 5300 DPPs were attributable to statin use and approximately 2100 DPPs to population-wide changes. Statins prevented almost $50 \%$ more deaths in the most affluent quintile compared with the most deprived. Conversely, population-wide changes in cholesterol prevented threefold more deaths in the most deprived quintile compared with the most affluent.

Conclusions: Population-wide secular changes in systolic blood pressure (SBP) and cholesterol levels helped to substantially reduce $\mathrm{CHD}$ mortality and the associated socioeconomic disparities. Mortality reductions were, in absolute terms, greatest in the most deprived quintiles, mainly reflecting their bigger

\section{Strengths and limitations of this study}

- This is the first IMPACT model to quantify the contributions of population risk factors and primary prevention treatments to recent changes in $\mathrm{CHD}$ mortality rates by socioeconomic quintiles.

- The datasets used for the model are representative of the English population and used deprivation scores for area of residence as an acceptable proxy indicator for socioeconomic status.

- Unlike the previous IMPACTSEC models, our study stratifies the analysis and results by gender. This allowed us to gain valuable new insights, for example the change in uptake levels for women in the least deprived quintile was almost as effective as the population-wide changes in SBP and cholesterol.

- We assumed that changes in the risk factors and treatment uptakes have equal effect across socioeconomic groups.

- The model was not able to explain around $14 \%$ of the total CHD mortality fall. One possible contributor might be the exclusion of other 'upstream' cardiovascular risk factors, which might affect SES groups differentially.

initial burden of disease. Statins for high-risk individuals also made an important contribution but maintained socioeconomic inequalities. Our results strengthen the case for greater emphasis on preventive approaches, particularly population-based policies to reduce SBP and cholesterol.

\section{INTRODUCTION}

The UK, as many other industrialised countries, has experienced a remarkable $60 \%$ reduction in coronary heart disease (CHD) mortality since the 1970s. However, CHD remains the leading cause of premature death. ${ }^{1}$ 
Approximately one-third of this initial CHD mortality reduction was attributable to treatments, and two-thirds to reductions in major risk factors. The biggest contributions came from a large decline in smoking prevalence since the 1960s and more recent reductions in blood pressure and cholesterol. ${ }^{2} 3$

The CHD mortality declines have demonstrated a changing relationship with socioeconomic status (SES). ${ }^{4-6}$ Initially it demonstrated a positive relationship with SES (ie, with affluence). ${ }^{7}$ However, this has now reversed in more recent studies in the UK, US, New Zealand, Australia and Scandinavia. ${ }^{8-10}$

Risk factors have also demonstrated strong socioeconomic patterning. Substantial positive associations between lower SES and higher smoking prevalence and higher blood pressure levels have been reported in several studies. ${ }^{11-13}$ However, for cholesterol, the evidence has been less dramatic, with a higher intake of saturated fats among the more deprived populations reported in most studies, ${ }^{14-16}$ but not all. ${ }^{17-19}$ Socioeconomic differences in both risk factors may thus explain some of the CHD mortality gradients. Thus, any attempt to reduce the CHD burden and tackle the associated socioeconomic inequalities should explicitly consider these major risk factors. ${ }^{20}$

Primary prevention medications to lower blood pressure and cholesterol therefore, have been a standard UK health policy for almost two decades. However, while their quantitative benefits to whole populations are accepted, their potential contributions to reduce inequalities are less clear. ${ }^{7}$ 21-25

The aim of this study was, therefore, to analyse the recent falls in CHD mortality and quantify the relative contributions from preventive medications and from population-wide changes in blood pressure and cholesterol levels, particularly exploring the potential effects on different socioeconomic groups.

\section{METHODS}

We used an extended version of the well-known IMPACT model to estimate the contributions of population-level risk factor changes and changes in treatment uptake on the CHD mortality decline in England between 2000 and 2007 for adults aged 25 and above, for two major risk factors-blood pressure and cholesterol. ${ }^{10}$

The IMPACT model applies the relative risk reduction quantified in previous randomised controlled trials (RCT) and meta-analyses to estimate the mortality reduction attributable to (1) temporal change in risk factor prevalence and (2) net change over the period in the uptake of specific treatments in patients with each specific form of CHD. This previously validated deterministic cell-based model has been described in detail elsewhere. ${ }^{21} 26$

The extended version IMPACT $\mathrm{SEC}_{\text {model }}{ }^{2}$ includes all the major CHD risk factors: smoking, systolic blood pressure (SBP), total cholesterol, body mass index (BMI), diabetes, physical inactivity and fruit and vegetable consumption. It also includes 45 medical and surgical treatments employed in nine different patient groups. Additionally, the model allows exploring the variation in CHD mortality trends by socioeconomic circumstances. Model inputs and outputs are stratified by the Index of Multiple Deprivation (IMD) quintiles as a proxy indicator of SES. ${ }^{14}$

Our primary outcome measure was the mortality fall or more specifically, the total number of deaths prevented or postponed (DPPs), for each deprivation quintile, that can be attributed to either population-level risk factor changes in SBP and cholesterol, or changes in the uptake of antihypertensive and dyslipidaemia treatments. The DPPs in 2007 relative to 2000 are defined as the difference between the number of CHD expected deaths in 2007 (had age, sex and SES quintile-specific CHD mortality rates in 2000 remained unchanged) and the observed figures.

To calculate the expected number of CHD deaths in 2007, we multiplied the age-sex-IMD quintile specific mortality rates from CHD in 2000 by the population counts for 2007 in that age-sex-IMD quintile stratum. Summing over all strata then yielded the expected number of deaths in 2007 had mortality rates that remained unchanged. Population counts, CHD mortality rates and observed number of deaths used in this step, along with sources, are enlisted in sections 3.1 and 3.2 of the online supplementary technical appendix.

The first part of the IMPACT $_{\mathrm{SEC}}$ model calculates the net benefit of statins and antihypertensive treatment in 2007. First, we calculated the expected number of DPPs if statin and antihypertensive uptake rates in 2000 remained constant by multiplying the 2000 age-sex-IMD quintile specific treatment uptake levels by the population counts for 2000 in that age-sex-IMD quintile stratum, the 1-year case fatality rate and the relative reduction in the case fatality rate as a result of the administered treatment. We did the same for the expected number of DPPs in 2007 by using the 2007 age-sex-IMD quintile specific treatment uptake levels. The difference between the expected number of DPPs (ie, using the treatment uptake rates in 2000) and the estimated number DPPs (ie, using the 2007 uptake rates) is the net benefit of treatments in 2007 .

The uptake levels for antihypertensives and statins were defined as the prevalence of never having had angina or heart attack, and currently taking medication specifically prescribed to treat high-blood pressure or lipid-lowering treatment. Treatment uptake values, estimates of treatment efficacy (relative risk reductions) and age-sex specific case fatality rates, along with their sources, are presented in sections 3.3-3.6 of the online supplementary technical appendix.

The second part of the IMPACT SEC $_{\text {model estimates }}$ the number of DPPs related to changes in SBP and cholesterol levels in the population. To calculate DPPs from changes in risk factors we used the regression approach, 
where the number of CHD deaths in 2000 were multiplied by the absolute change in risk factor level (absolute difference in the risk factors levels between 2000 and 2007) and by a regression $\beta$-coefficient quantifying the estimated relative change in CHD mortality that would result from a one unit change in risk factor level. Risk factors mean levels and $\beta$-coefficients, along with their sources, are presented in sections $3.7-3.9$ of the online supplementary technical appendix.

Recent reductions in CHD mortality have been the result of simultaneous change in multiple risk factors. Hence, part of the effect of one risk factor may be mediated through another. In this regard, we used a cumulative risk reduction adjustment factor (AF) to adjust downwards the DPPs attributed to multiple risk factors acting additively or separately, more details can be found in section 2.5 of the online supplementary technical appendix.

Also we considered that some overlap between pharmacological and non-pharmacological contributions to risk factor DPPs occur. Therefore, to estimate the impact of population-wide reduction in total cholesterol due to non-pharmacological change only, we subtracted the estimated effect of cholesterol-lowering treatments uptake levels change from the overall number of DPPs due to change in mean total cholesterol. A similar procedure was carried out for SBP and antihypertensive treatments. For more details see section 2.6 of the online supplementary technical appendix.

Finally, we implemented sensitivity analysis using the EXCEL add-in Ersatz software which allows Monte Carlo simulation. This allows us to calculate $95 \%$ uncertainty intervals (95\% UI) for all outputs, based on 5000 draws from specified probabilistic distributions for the model input variables. The probabilistic distributions and their parameters used for the each of the input variables can be found in section 2.8 of the online supplementary technical appendix.

More details on the methodology and worked examples can be found in the online supplementary technical appendix.

\section{RESULTS}

\section{SBP and cholesterol population levels}

Figure 1 depicts the trends in population SBP and cholesterol levels between 2000 and 2007, stratified by IMD quintiles and sex. SBP fell substantially between 2000 and 2007 , by an average of $5.4 \mathrm{~mm} \mathrm{Hg}$ in women and by $2.5 \mathrm{~mm} \mathrm{Hg}$ in men. Total cholesterol also fell substantially (by approximately $0.20 \mathrm{mmol} / \mathrm{L}$ ), but equally in men and women.

There was no evidence of a social gradient, since the population factors levels were similar across IMD quintiles with no statistically significant difference between them.

\section{Antihypertensive and statin treatment uptakes}

Figure 2 depicts treatments uptakes between 2000 and 2007: there was a substantial increase in both treatment uptakes, especially statins. Uptake levels of antihypertensive treatments and statins were remarkably equitable across quintiles for men and women, with no statistically significant differences between them.

\section{Deaths prevented or postponed}

There were approximately 38000 fewer CHD deaths in 2007 than if 2000 mortality rates had persisted and been applied to 2007 population estimates for England. Our model was able to explain approximately 32800 (86.3\%) of these fewer deaths (see table 1). Approximately 7100 (95\% UI, 3500-14 200) fewer deaths (19\% of the total mortality reduction) were attributed to increases in the uptake levels of treatments for high-blood pressure and raised cholesterol. Approximately 13300 (8500-17 400) DPPs (35\% of the mortality reduction) were attributed to population falls in blood pressure and cholesterol in asymptomatic individuals after subtracting the estimated effect of increases in treatment uptakes. The remaining $32 \%$ of the deaths prevented or postponed in our model were attributed to other risk factors and treatments.

Figure 3 shows the number of deaths prevented or postponed from changes in the population mean levels of SBP and cholesterol (figure 3A, left panel) and from changes in the treatments uptake levels (figure $3 \mathrm{~B}$, right panel). We can highlight some key aspects:

(1) Population falls in SBP and cholesterol resulted in more DPPs than increases in uptake levels changes of antihypertensives and statins; (2) Most of the mortality reduction through population changes reflected falls in SBP rather than in cholesterol; (3) By contrast, most of the effect of treatment uptake levels changes was through increments in the uptake levels in statin use rather than antihypertensive use, reflecting the larger increase in statins use during the period of study (e.g., statin uptake rate in 2000 was around $1 \%$ compared to $12 \%$ in 2007); (4) Substantial numbers of DPPs were observed in all social class groups; (5) The absolute effect of population changes on DPPs was larger among persons residing in the most deprived quintiles; and (6) by contrast, the number of DPPs attributable to increases in treatment uptake levels was remarkably equitable across SES groups. However, statin uptakes apparently postponed or prevented slightly more deaths in the most affluent quintile than in the most deprived quintile (figure 3B).

\section{Systolic blood pressure}

Overall, SBP falls between 2000 and 2007 prevented or postponed approximately 13000 (8100-17 500) deaths (34.2\% of the total mortality reduction). Approximately $1800(700-3900)$ of those were attributable to antihypertensive treatments $(4.7 \%$ of the total mortality reduction) and some 11200 DPPs (6500-15 100), over sixfold more, were attributable to population-wide SBP changes (29.5\% of the total mortality reduction). Substantially more DPPs through population-wide changes occurred in the most deprived quintile: 2400 (1600-3100) 
Cholesterol population-levels for

Men

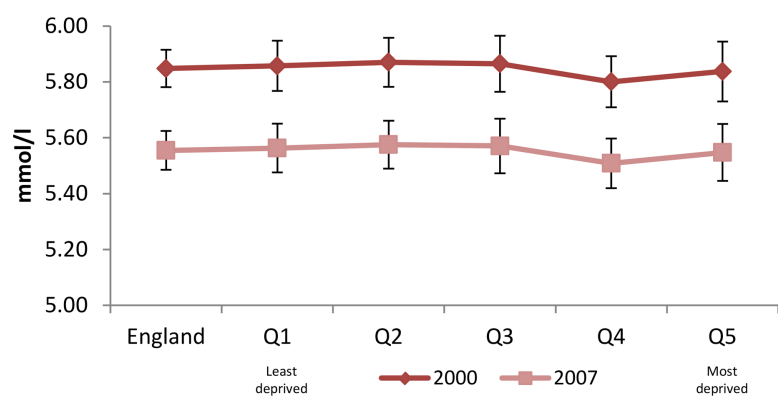

SBP population-levels for Men

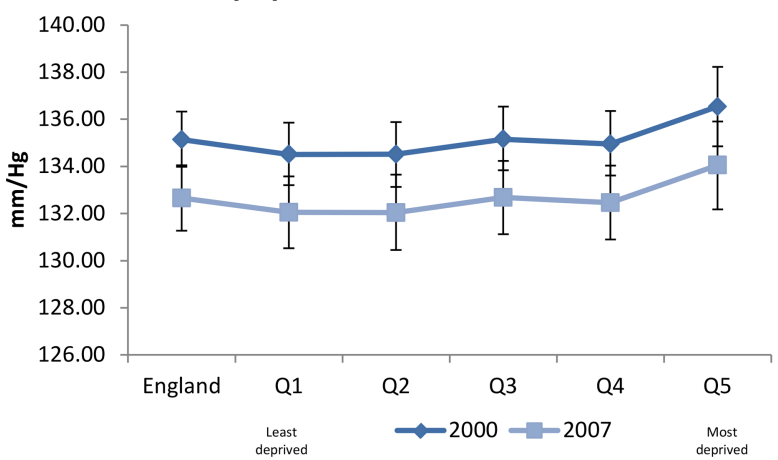

Cholesterol population-levels for Women

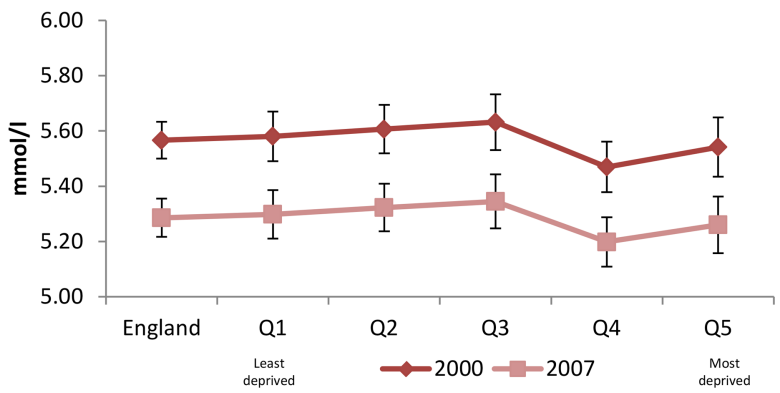

SBP population-levels for Women

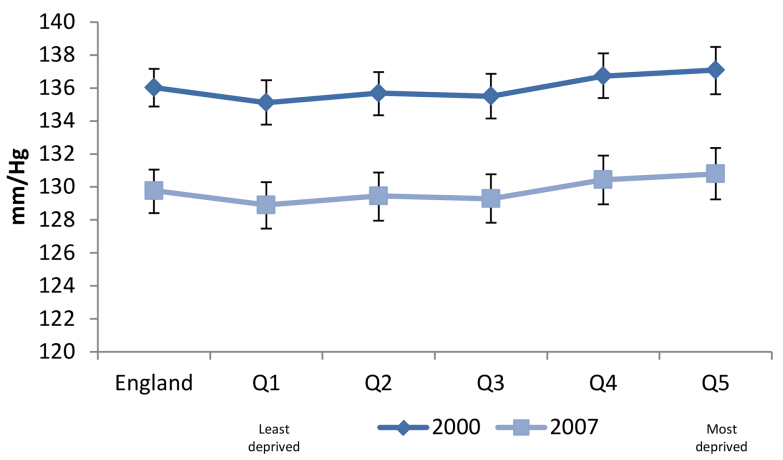

Figure 1 Mean values of systolic blood pressure (SBP) and cholesterol (with 95\% UI) between 2000 and 2007 for England stratified by deprivation quintiles and sex.

compared with the most affluent quintiles: 1800 (1000-2600). Thus population-wide changes apparently helped to reduce inequalities in absolute terms.
Conversely, changes in treatment uptake levels demonstrated the opposite effect, since more deaths were prevented in the most affluent quintile (360 DPPs)
Anti-hypertensive uptake levels for

Men

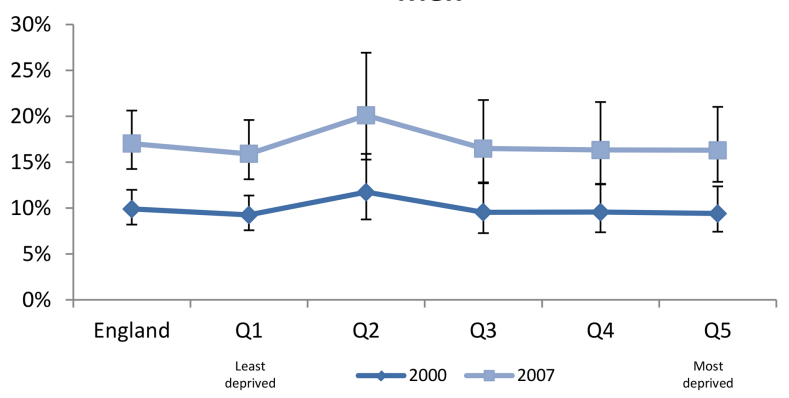

Statins uptake levels for Men

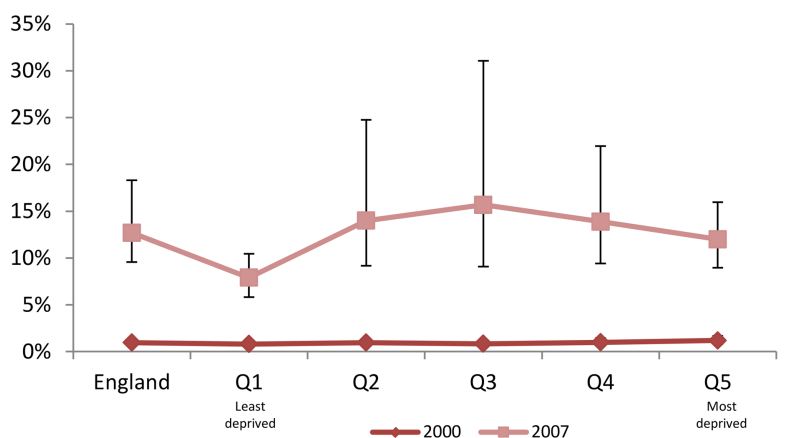

Anti-hypertensive uptake levels for Women

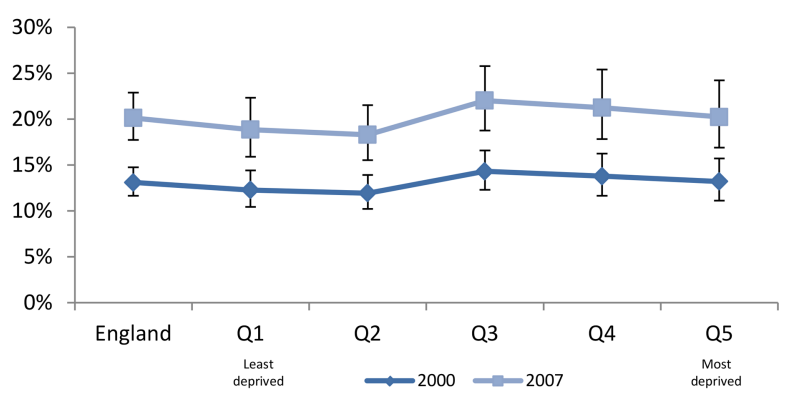

Statins uptake levels for Women

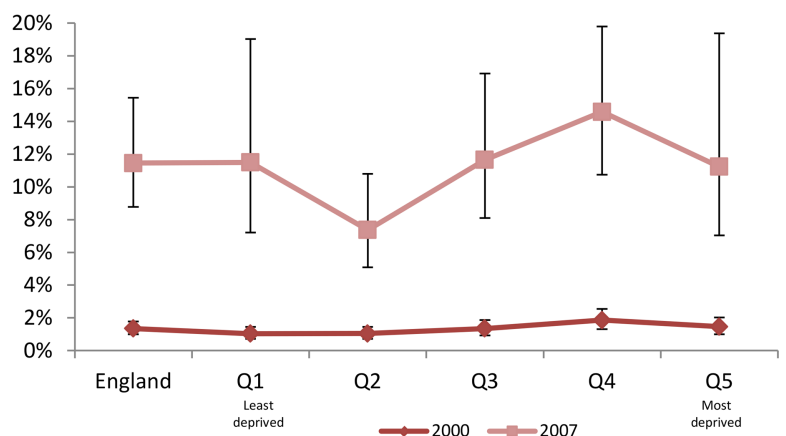

Figure 2 Uptake levels and proportion change in treatment uptake (with 95\% UI) between 2000 and 2007 for England stratified by deprivation quintiles and sex. 
Table 1 CHD deaths prevented or postponed between 2000 and 2007 in England, stratified by deprivation quintiles

Deaths prevented or postponed (DPP)

\begin{tabular}{lllllll}
\hline & England & $\begin{array}{l}\text { IMD quintile 1 } \\
\text { affluent }\end{array}$ & IMD quintile 2 & IMD quintile 3 & IMD quintile 4 & $\begin{array}{l}\text { IMD quintile 5 } \\
\text { deprived }\end{array}$ \\
\hline Mean & 32770 & 5775 & 6745 & 7015 & 6870 & 6370 \\
$95 \%$ LL & 25990 & 4430 & 5320 & 5420 & 5400 & 5100 \\
$95 \%$ UL & 41550 & 7705 & 8515 & 9360 & 8765 & 7830 \\
\hline
\end{tabular}

compared to the most deprived (280 DPPs). However, in both cases, SES differences were not statistically significant. Detailed outputs with uncertainty intervals can be found in section 4 of the online supplementary technical appendix.

\section{Cholesterol}

Overall, cholesterol falls between 2000 and 2007 resulted in approximately 7400 (3900-14500) fewer deaths (19.5\% of the total mortality reduction). This total comprised some 5300 (2100-12 300) fewer deaths (13.9\% of the total mortality reduction) attributable to statin medications and approximately 2100 (1000-3200) fewer deaths $(5.5 \%$ of the total mortality reduction) attributable to population-wide falls in cholesterol. Statin medications prevented some 1100 (400-2700) deaths in the most affluent quintile compared to approximately 800 (300-1900) DPPs in the most deprived quintile. Conversely, population changes in cholesterol resulted in approximately 700 (500-1000) DPPs in the most deprived quintile and some 200 (40-400) DPPs in the most affluent quintile. However, like SBP, there was no clear SES gradient. Section 4 of the online supplementary technical appendix provides detailed outputs with uncertainty intervals.

\section{Gender differences}

Figure 4 shows the number of deaths prevented or postponed in men and women from falls in the population mean levels of SBP and cholesterol (figure 4A, left panels) and from increases in the treatment uptake levels (figure 4B, right panels). For men, although most of the mortality reduction came from population falls in SBP, cholesterol reductions also had a considerable larger effect in reducing mortality compared to women (four times higher). By contrast, the number of DPPs due to increases in treatment uptakes in men appeared remarkably equitable across SES groups.

For women, the impressive reduction in SBP mean level between 2000 and 2007 contributed the most to the total mortality reduction and in all quintiles, whereas population level reductions of cholesterol had a

B
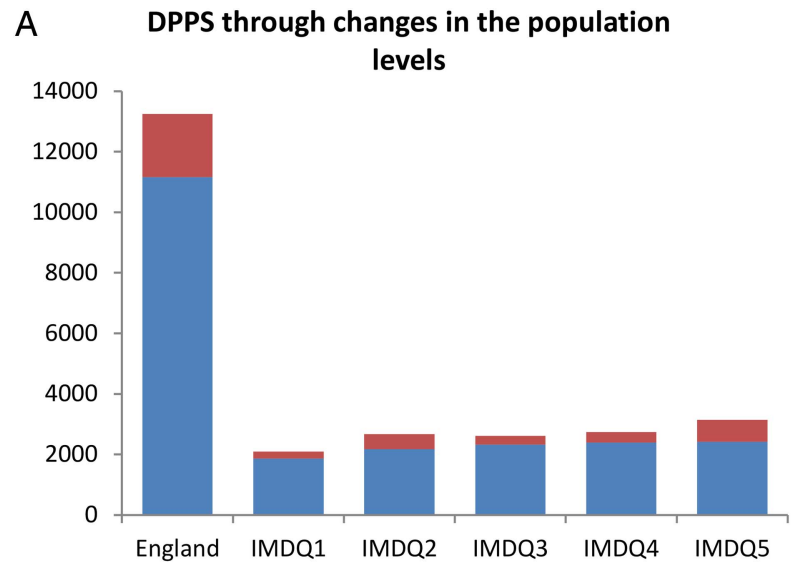

\begin{tabular}{|c|c|c|c|c|c|c|}
\hline DPPs & England & $\begin{array}{c}\text { IMD } \\
\mathbf{1}\end{array}$ & $\begin{array}{c}\text { IMD } \\
\mathbf{2}\end{array}$ & $\begin{array}{c}\text { IMD } \\
\mathbf{3}\end{array}$ & $\begin{array}{c}\text { IMD } \\
\mathbf{4}\end{array}$ & $\begin{array}{c}\text { IMD } \\
\mathbf{5}\end{array}$ \\
\hline Total & 13253 & 2093 & 2666 & 2608 & 2742 & 3143 \\
\hline SBP & 11162 & 1861 & 2168 & 2321 & 2391 & 2421 \\
\hline Cholesterol & 2091 & 232 & 498 & 287 & 351 & 722 \\
\hline
\end{tabular}

B DPS through changes in the treatments uptakes

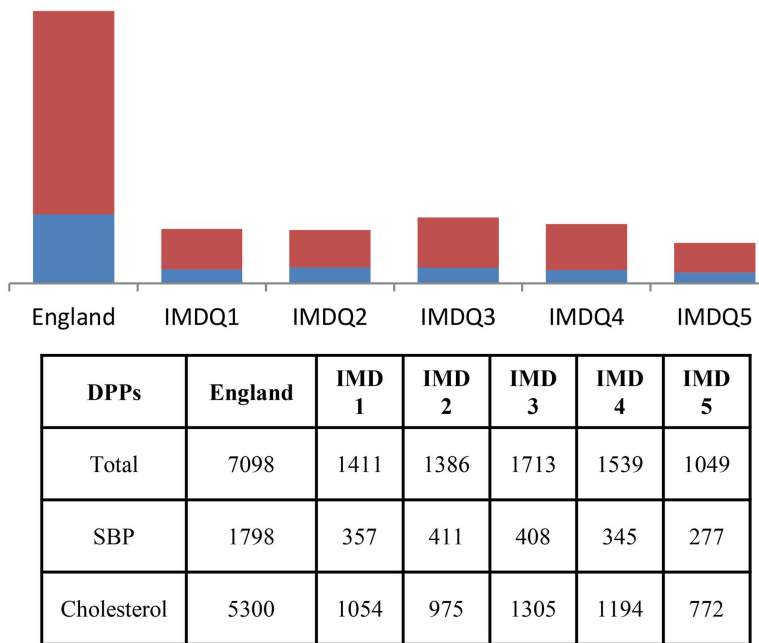

Figure 3 Number of deaths prevented or postponed (DPPs) between 2000 and 2007 in England, attributable to changes in the population in SBP and cholesterol (A, left panel), changes in uptakes levels for antihypertensive treatments and statins (B, right panel); stratified by deprivation quintiles 
A

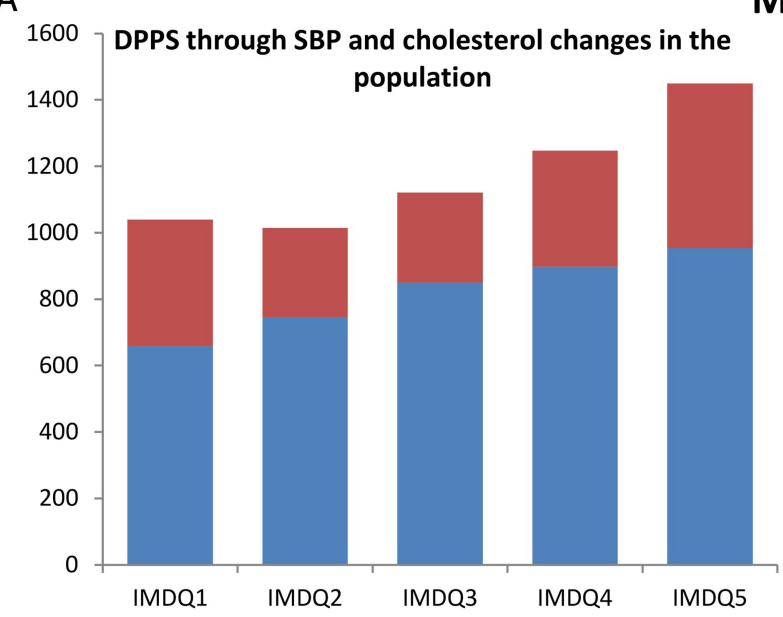

MEN

DPPS through changes in the treatments uptakes

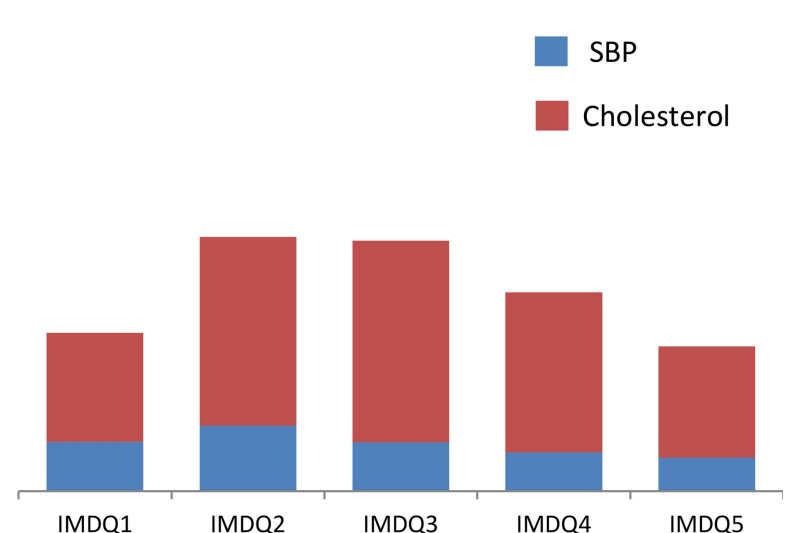

WOMEN

B

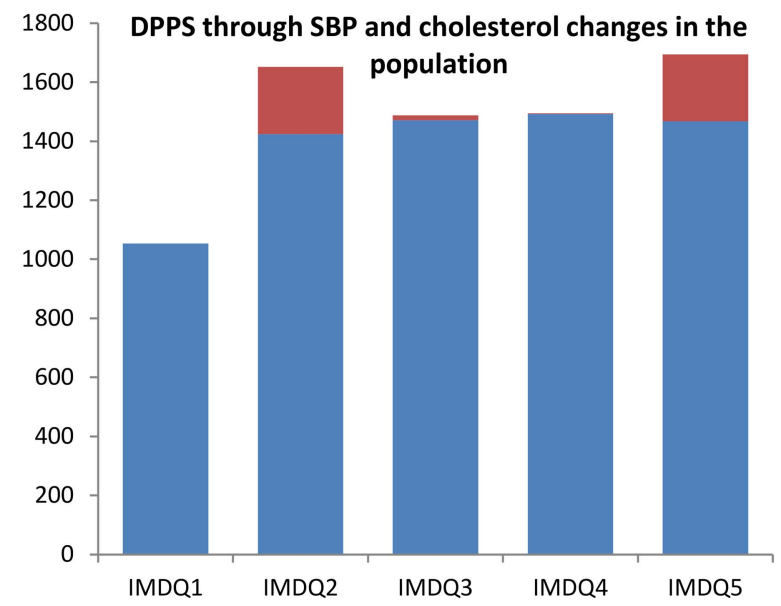

DPPS through changes in the treatments uptakes

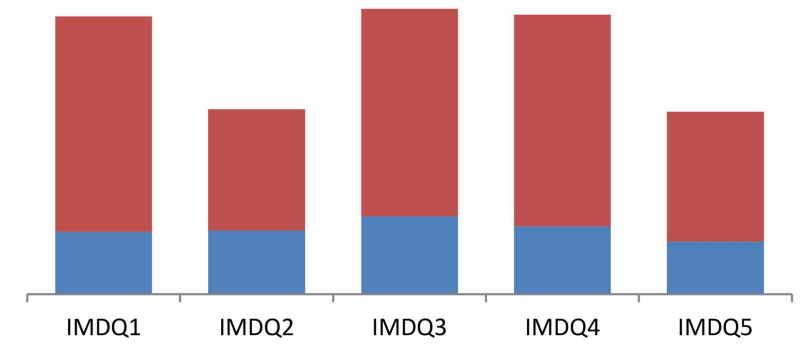

Figure 4 (A and B) Number of deaths prevented or postponed (DPPs) from changes in the population in systolic blood pressure (SBP) and cholesterol, changes in uptakes levels for antihypertension and statins between 2000 and 2007 in England, stratified by deprivation quintiles and sex.

smaller benefit. Moreover, the joint benefit of increasing treatment uptakes (antihypertensive and statins) in women appeared to have an important effect: for example, in the most affluent quintile (IMDQ1) the reduction in DPPs due to the increase in treatment uptakes for women was almost as effective as the population-wide falls in both sexes for that quintile.

However, in terms of differences between men and women, the results of the uncertainty analysis suggest that these are not significant in statistical terms. More detailed outputs split by gender can be found in section 5 of the online supplementary technical appendix.

\section{DISCUSSION}

CHD mortality in England fell by a remarkable 34\% between 2000 and 2007. This represents an impressive 38000 fewer deaths from CHD in 2007 than if the 2000 mortality rates had persisted. Reductions in major cardiovascular risk factors of blood pressure and cholesterol explained for almost two-thirds of this large mortality fall.

\section{Blood pressure trends}

Declines in the population blood pressure level made the largest contribution to the overall fall in CHD mortality. In contrast, antihypertensive treatments produced only modest benefits. First, because the baseline CHD event rate was low in asymptomatic individuals $(\leq 1 \%$ per year) yielding only a small reduction of the attributable risk during the period of study. ${ }^{27}$ Second, treatment efficacy is low; and third, blood pressure control is still poor (adherence levels to medication are around 60\%), leading in conjunction to a substantial residual risk. ${ }^{21} 28$

\section{Cholesterol trends}

Population-wide falls in cholesterol levels averted more deaths in the most deprived quintiles, reflecting similar absolute falls but much higher baseline mortality rates. The increase in the uptake of statins between 2000 and 2007 made an even greater contribution to the overall mortality fall: twofold greater than the change in population cholesterol ( $16 \%$ vs $6 \%$ ), and with equitable benefits across all five SES groups. 


\section{Comparisons with other studies}

Our results are consistent with previous analyses in the UK and around the world, supporting the importance of this study beyond England. Using the IMPACT model to examine contributions to the overall reductions in CHD mortality in England and Wales population between 1981 and 2000, Unal, Critchley ${ }^{3}$ reported a higher contribution from blood pressure changes (compared to cholesterol). Some $76 \%$ of this contribution was attributable to population-wide changes rather than antihypertensive medications. IMPACT analyses carried out in the USA and Irish populations between 19802000 and 1985-2000 likewise observed substantially greater benefits attributable to secular changes in risk factors rather than treatments. ${ }^{26} 27$

The analysis by DeWilde, Carey ${ }^{29}$ suggested that reported blood pressure treatments were responsible for $25 \%$ of $5 \mathrm{~mm} \mathrm{Hg}$ reduction in SBP during the period 1994-2009 for England.

Emberson $e t a l^{30}$ applied a very different methodology using evidence from randomised control trials and cohort studies to analyse the effectiveness of populationwide changes in risk factor levels against the high-risk individual approach. Their findings were entirely consistent with ours. They concluded that a mere $10 \%$ reduction in population-wide blood pressure and cholesterol levels might achieve a $45 \%$ reduction in cardiac events in the long term. However, it would be needed to provide treatment to approximately $26 \%$ of the UK population at high risk to achieve only a $34 \%$ reduction in cardiac events. The US CHD policy model likewise reported that population-wide reductions of salt intake $(3 \mathrm{~g} /$ day $)$ might prevent between 44000 and 90000 deaths. ${ }^{31}$

\section{Strengths and limitations}

This is the first IMPACT model to quantify the contributions of population risk factors and primary prevention treatments to recent changes in CHD mortality rates by socioeconomic quintiles.

The data sets used for the model are representative of the English population and used deprivation scores for area of residence as an acceptable proxy indicator for socioeconomic status. This allowed a sufficient sample size to quantify the effect of risk factor modification through changes in population-wide risk factor levels and treatment uptakes.

Unlike, the previous IMPACT SEC $_{\text {models (Bajekal } e t a l^{2}}$ and Scholes $e t a l^{22}$ ), our study stratifies the analysis and results by gender. This allowed us to gain valuable new insights. For example, changes in SBP and cholesterol population levels for women led to the highest number of DPPs for all quintiles. More surprisingly, the change in uptake levels for women in the least deprived quintile was almost as effective as the population-wide changes in SBP and cholesterol. This suggests that any attempt to tackle the socioeconomic inequalities in CHD mortality should explicitly consider these gender differences.
However, our study has limitations that should also be acknowledged. First, the area-level categorisation may not be representative of individual circumstances. Furthermore, observed SES differences in CHD mortality might reflect not material deprivation but other confounding and mediator factors such as alcohol consumption, obesity or ethnicity. However, the IMD is a comprehensive multidimensional construct of socioeconomic status made up of seven domains, and based on small geographical areas (less than 1500 residents) called lower level super output areas (LSOAs). The advantage of using LSOAs is that their smaller geographical size also allows for a more detailed knowledge of deprived areas.

Our risk factor effect data might still have some residual confounding. Statins and antihypertensive medication data are from the surveys; therefore, some misclassification bias might be present.

We assumed that treatments and lifestyle changes have an immediate effect on CHD mortality, which might not be entirely true. However, Capewell and O'Flaherty ${ }^{23} 32$ pointed out evidence from clinical trials and policy interventions which consistently suggest that changes in diet and lifestyle across entire populations can be rapidly followed by dramatic declines in mortality.

We assumed that changes in the risk factors and treatment uptakes have equal effect across socioeconomic groups. However, the benefits of falls in risk factors or increases in treatment uptakes may be higher in more affluent groups. ${ }^{2}$ This may partly explain the faster rates of CHD mortality decline in the most affluent quintiles as Bajekal $e t a l^{10}$ pointed out. Likewise, we assumed that the relative risk reduction due to treatments remained constant from 2000 to 2007.

We simply subtracted the mortality gains from increasing uptake levels of statins from the overall gains due to reductions in total cholesterol to estimate the impact of population-wide reduction in total cholesterol due to non-pharmacological change only. This mutually exclusive adjudication of cause adjustment might overestimate medication benefit.

Given the background of higher mortality and morbidity in the more deprived quintiles, DPPs might overestimate the actual health gain, as we do not know the additional life span gained by preventing a specific death at a specific time. This might result in a lesser reduction in inequalities than DPPs alone would suggest.

Finally, our model was not able to explain around $14 \%$ of the total CHD mortality fall between 2000 and 2007. One possible contributor might be the exclusion of other 'upstream' cardiovascular risk factors, which might affect SES groups differentially, for example, psychosocial stress. ${ }^{33}$

\section{Implications for public health and clinical care}

This study shows that population-wide secular falls in blood pressure and cholesterol have substantially helped to decrease CHD mortality and reduce the associated 
socioeconomic disparities in absolute terms. Furthermore, as we discussed earlier, there is an increasing body of evidence to support the use of population-wide approaches to reduce CHD risk factors. Mackenbach $e t a b^{34}$ recently evaluated 22 successful preventive interventions in the Netherlands. Approximately $75 \%$ of the health gains during the period 1970-2010 were achieved by a population approach and just $25 \%$ by a high-risk individual approach.

In the UK, the population-wide fall in blood pressure is consistent with the recent successful implementation of policies to reduce salt intake. Similar trends have been reported in other developed countries. ${ }^{21}{ }^{28}$ There are also several international examples where policy interventions have proven to be effective at achieving significant reductions in saturated fats, trans-fats and calories in processed foods and takeaway meals. ${ }^{24} 313536$ However, policies to reduce saturated fats and trans-fats have so far been neglected in the UK. ${ }^{25}$

Conversely, targeting high-risk individuals with medication appears less effective and may also widen socioeconomic inequalities in CHD mortality. ${ }^{37} 38$ Any intervention that requires people to mobilise their own resources (material and psychological) will understandably favour those who have greater resources ${ }^{37}$ and thus, widen social inequalities. Thus, those with the poorest health will benefit the least from such interventions. ${ }^{38}$

However, there is no simple choice between either population-based or high-risk strategies to reduce CHD mortality. The approaches are complementary in delivering the greatest public health benefit. ${ }^{39} 40$ It is, however, clear that individual-based treatment strategies can afford only modest reductions in mortality compared with addressing risk factors population wide.

Severely limited healthcare budgets are now forcing planning systems to consider how best to allocate future resources. Our results strengthen the case for greater emphasis on preventive approaches, particularly population-based policies to reduce blood pressure and cholesterol. Such strategies might be more powerful, rapid, cost-effective and equitable than additional preventive medications. ${ }^{25}$

\section{Author affiliations}

${ }^{1}$ Department of Public Health and Policy, University of Liverpool, Liverpool, UK

${ }^{2}$ Division of Cardiology, University of British Columbia, Vancouver, Canada

${ }^{3}$ Health and Social Surveys Research Group, University College London, UK

${ }^{4}$ Global Public Health, University of Chester, Chester, UK

${ }^{5}$ Public Health Wales, Swansea, UK

Acknowledgements The authors would like to thank Rosalind Raine and Madhavi Bajekal for their work in developing the original IMPACT SEC $_{\text {model. }}$

Collaborators Rosalind Raine and Madhavi Bajekal.

Contributors MGC drafted the manuscript, analysed the results and conducted the uncertainty analysis in collaboration with SC, MOF and RA. RA conducted the initial literature review and initial set up of the model in collaboration with SC. NH, SS, EW and JL contributed to the interpretation of the results and to the drafting and finalisation of the manuscript.
Funding MGC, MOF and SC are in part funded by the NIHR School of Public Health Research (grant SPHR-LIL-PH1-MCD). RA was funded by the Liverpool PCT FSF scheme.

Competing interests None.

Provenance and peer review Not commissioned; externally peer reviewed.

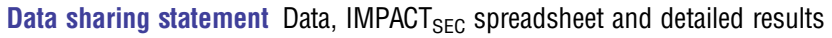
are available on request by emailing Maria Guzman-Castillo.

Open Access This is an Open Access article distributed in accordance with the Creative Commons Attribution Non Commercial (CC BY-NC 4.0) license, which permits others to distribute, remix, adapt, build upon this work noncommercially, and license their derivative works on different terms, provided the original work is properly cited and the use is non-commercial. See: http:// creativecommons.org/licenses/by-nc/4.0/

\section{REFERENCES}

1. Murray CJL, Richards MA, Newton JN, et al. UK health performance: findings of the Global Burden of Disease Study 2010. Lancet 2013;381:997-1020.

2. Bajekal M, Scholes $\mathrm{S}$, Love $\mathrm{H}$, et al. Analysing recent socioeconomic trends in coronary heart disease mortality in England, 2000-2007: a population modelling study. PLOS Med 2012;9:e1001237.

3. Unal B, Critchley JA, Capewell S. Explaining the decline in coronary heart disease mortality in England and Wales between 1981 and 2000. Circulation 2004;109:1101-7.

4. Kraus JF, Borhani NO, Franti CE. Socioeconomic status, ethnicity, and risk of coronary heart disease. Am J Epidemiol 1980;111:407-14.

5. Pocock SJ, Cook DG, Shaper AG, et al. Social class differences in ischaemic heart disease in British men. Lancet 1987;330:197-201.

6. Marmot M, Theorell T. Social class and cardiovascular disease: the contribution of work. Int J Health Serv 1988;18:659-74.

7. Cassel J, Heyden S, Bartel AG, et al. Incidence of coronary heart disease by ethnic group, social class, and sex. Arch Intern Med 1971;128:901.

8. Hemingway $\mathrm{H}$, Shipley $\mathrm{M}$, Macfarlane $\mathrm{P}$, et al. Impact of socioeconomic status on coronary mortality in people with symptoms, electrocardiographic abnormalities, both or neither: the original Whitehall study 25 year follow up. J Epidemiol Community Health 2000;54:510-16.

9. Salomaa V, Niemelä M, Miettinen $\mathrm{H}$, et al. Relationship of socioeconomic status to the incidence and prehospital, 28-day, and 1 -year mortality rates of acute coronary events in the FINMONICA Myocardial Infarction Register Study. Circulation 2000;101:1913-18.

10. Bajekal M, Scholes S, O'Flaherty M, et al. Unequal trends in coronary heart disease mortality by socioeconomic circumstances, England 1982-2006: an analytical study. PloS ONE 2013;8:e59608.

11. Dressler WW. Education, lifestyle and arterial blood pressure. J Psychosom Res 1990;34:515-23.

12. Qureshi Al, Suri MFK, Kirmani JF, et al. Prevalence and trends of prehypertension and hypertension in United States: National Health and Nutrition Examination Surveys 1976 to 2000. Med Sci Monit 2005;11:CR403-9.

13. Hoeymans $\mathrm{N}$, Smit HA, Verkleij $\mathrm{H}$, et al. Cardiovascular risk factors in relation to educational level in 36000 men and women in The Netherlands. Eur Heart $J$ 1996;17:518-25.

14. Marmot MG, Syme SL, Kagan A, et al. Epidemiologic studies of coronary heart disease and stroke in Japanese men living in Japan, Hawaii and California: prevalence of coronary and hypertensive heart disease and associated risk factors. Am J Epidemiol 1975;102:514-25.

15. Lindström M, Hanson BS, Brunner E, et al. Socioeconomic differences in fat intake in a middle-aged population: report from the Malmö Diet and Cancer Study. Int J Epidemiol 2000;29:438-48.

16. Johansson L, Thelle DS, Solvoll K, et al. Healthy dietary habits in relation to social determinants and lifestyle factors. Br J Nutr 1999;81:211-20.

17. Bolton-Smith C, Smith WCS, Woodward M, et al. Nutrient intakes of different social-class groups: results from the Scottish Heart Health Study (SHHS). Br J Nutr 1991;65:321-35

18. Hulshof $\mathrm{K}$, Brussaard JH, Kruizinga AG, et al. Socio-economic status, dietary intake and 10 y trends: the Dutch National Food Consumption Survey. Eur J Clin Nutr 2003;57:128-37.

19. Dubois L, Girard M. Social position and nutrition: a gradient relationship in Canada and the USA. Eur J Clin Nutr 2001;55:366-73. 
20. Tobias M, Rodgers A. Can we eliminate inequalities in coronary disease? Absolutely. Lancet 372:1612-13.

21. Capewell S, Morrison CE, McMurray JJ. Contribution of modern cardiovascular treatment and risk factor changes to the decline in coronary heart disease mortality in Scotland between 1975 and 1994. Heart 1999;81:380-6.

22. Scholes S, Bajekal M, Norman P, et al. Quantifying policy options for reducing future coronary heart disease mortality in England: a modelling study. PloS ONE 2013;8:e69935.

23. Capewell S, O'Flaherty M. Rapid mortality falls after risk-factor changes in populations. Lancet 2011;378:752-3.

24. Glantz S, Gonzalez M. Effective tobacco control is key to rapid progress in reduction of non-communicable diseases. Lancet 2012;379:1269-71.

25. NICE. Public Health Guidance 25: Prevention of cardiovascular disease at population level [cited 201301 December].

26. Young F, Capewell S, Ford ES, et al. Coronary mortality declines in the US between 1980 and 2000: quantifying the contributions from primary and secondary prevention. Am J Prev Med 2010;39:228-34.

27. Kabir Z, Bennett K, Shelley E, et al. Comparing primary prevention with secondary prevention to explain decreasing coronary heart disease death rates in Ireland, 1985-2000. BMC Public Health 2007;7:117.

28. Carroll MD, Lacher DA, Sorlie PD, et al. Trends in serum lipids and lipoproteins of adults, 1960-2002. JAMA 2005;294:1773-81.

29. DeWilde S, Carey I, Shah S, et al. Trends in blood pressure in England: good treatment or good luck? J Epidemiol Community Health 2012;66(Suppl 1):A41.

30. Emberson J, Whincup P, Morris R, et al. Evaluating the impact of population and high-risk strategies for the primary prevention of cardiovascular disease. Eur Heart J 2004;25:484-91.
31. Bibbins-Domingo K, Chertow GM, Coxson PG, et al. Projected effect of dietary salt reductions on future cardiovascular disease. $N$ Engl $J$ Med 2010;362:590-9.

32. Capewell S, Flaherty M. Can dietary changes rapidly decrease cardiovascular mortality rates? Eur Heart $J$ 2011;32:1187-9.

33. Harald K, Koskinen $\mathrm{S}$, Jousilahti $\mathrm{P}$, et al. Changes in traditional risk factors no longer explain time trends in cardiovascular mortality and its socioeconomic differences. J Epidemiol Community Health 2008;62:251-7.

34. Mackenbach JP, Lingsma HF, van Ravesteyn NT, et al. The population and high-risk approaches to prevention: quantitative estimates of their contribution to population health in the Netherlands, 1970-2010. Eur J Public Health 2013;23:909-15.

35. Zatonski WA, Willett W. Changes in dietary fat and declining coronary heart disease in Poland: population based study. BMJ 2005;331:187-8.

36. Cobiac LJ, Vos T, Veerman JL. Cost-effectiveness of interventions to reduce dietary salt intake. Heart 2010;96:1920-5.

37. Capewell S, Graham H. Will cardiovascular disease prevention widen health inequalities? PLoS Med 2010;7:e1000320.

38. White M, Adams J, Heywood P. How and why do interventions that increase health overall widen inequalities within populations. Soc Inequality Public Health 2009:65-82.

39. The Food Labelling Regulations 1996 [cited 201312 November] http://www.legislation.gov.uk/uksi/1996/1499/introduction/made

40. Singh GM, Danaei G, Pelizzari PM, et al. The age associations of blood pressure, cholesterol and glucose: analysis of health examination surveys from international populations. Circulation 2012;125:2204-11. 


\section{Correction}

Guzman-Castillo M, Ahmed R, Hawkins N, et al. The contribution of primary prevention medication and dietary change in coronary mortality reduction in England between 2000 and 2007: a modelling study. BMJ Open 2015;5:e006070.

(1) The Acknowledgement section should read:

We would like to thank Rosalind Raine and Madhavi Bajekal for their work in developing the original IMPACTSEC model. The English IMPACTsec project was conducted by Madhavi Bajekal (MB) and Shaun Scholes (SS). Hande Love set up the worksheet template; SS populated the model and was its custodian. MB ensured the integrity of inputs and outputs and provided SEC-related methodological solutions. Martin O'Flaherty \& Nat Hawkins provided support, clinical expertise and generated the therapeutic input. The UCL team was led by Rosalind Raine. Simon Capewell co-ordinated the overall project.

(2) The design section should read:

Design: Retrospective analysis using the IMPACTsec policy model.

(3) 'Strengths and limitations' and 'Summary' sections should read:

IMPACTsec is the first model to quantify the contributions of population risk factors and primary prevention treatments to recent changes in CHD mortality rates by socioeconomic quintiles.

(4) Appendix/References section has been replaced online to include a version that references Bajekal; no other changes have been made.

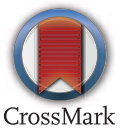

BMJ Open 2015;5:e006070corr1. doi:10.1136/bmjopen-2014-006070corr1 